\title{
Origin of ultra-compact dwarfs: a dynamical perspective
}

Hong-Xin Zhang ${ }^{1,2,3,4}$, Eric W. Peng ${ }^{1,2}$, Patrick Côté ${ }^{5}$, Chengze Liu $^{6,7}$, Laura Ferrarese $^{5}$, Jean-Charles Cuillandre ${ }^{8}$, Nelson Caldwell ${ }^{9}$, Stephen D. J. Gwyn ${ }^{5}$, Andrés Jordán ${ }^{10}$, Ariane Lançon ${ }^{11}$, Biao Li ${ }^{1,2}$, Roberto P. Muñoz ${ }^{10,11}$, Thomas H. Puzia ${ }^{10}$, Kenji Bekki ${ }^{12}$, John Blakeslee $^{5}$, Alessandro Boselli ${ }^{13}$, Michael J. Drinkwater ${ }^{14}$, Pierre-Alain Duc ${ }^{15}$, Patrick Durrell ${ }^{16}$, Eric Emsellem ${ }^{17,18}$, Peter Firth $^{14}$ and Ruben Sánchez-Janssen ${ }^{5}$

${ }^{1}$ Department of Astronomy, Peking University, Beijing 100871, China email: hongxin@pku.edu.cn

${ }^{2}$ Kavli Institute for Astronomy and Astrophysics, Peking University, Beijing 100871, China ${ }^{3}$ CAS-CONICYT Fellow

${ }^{4}$ Chinese Academy of Sciences South America Center for Astronomy, Camino EI Observatorio \#1515, Las Condes, Santiago, Chile

${ }^{5}$ National Research Council of Canada, Herzberg Astronomy and Astrophysics Program, 5071 West Saanich Road, Victoria, BC V9E 2E7, Canada

${ }^{6}$ Center for Astronomy and Astrophysics, Department of Physics and Astronomy, Shanghai Jiao Tong University, Shanghai 200240, China

${ }^{7}$ Shanghai Key Lab for Particle Physics and Cosmology, Shanghai Jiao Tong University, Shanghai 200240, China

${ }^{8}$ Canada-France-Hawaii Telescope Corporation, Kamuela, HI 96743, USA

${ }^{9}$ Harvard-Smithsonian Center for Astrophysics, Cambridge, MA, 02138

${ }^{10}$ Instituto de Astrofísica, Facultad de Física, Pontificia Universidad Católica de Chile, Av. Vicuña Mackenna 4860, 7820436 Macul, Santiago, Chile

${ }^{11}$ Observatoire astronomique de Strasbourg, Université de Strasbourg, CNRS, UMR 7550, 11 rue de l'Universite, F-67000 Strasbourg, France

${ }^{12}$ School of Physics, University of New South Wales, Sydney 2052, NSW, Australia

${ }^{13}$ Aix Marseille Université, CNRS, LAM (Laboratoire d'Astrophysique de Marseille) UMR 7326, F-13388 Marseille, France

${ }^{14}$ School of Mathematics and Physics, University of Queensland, Brisbane, QLD 4072, Australia

${ }^{15}$ Laboratoire AIM Paris-Saclay, CNRS/INSU, Université Paris Diderot, CEA/IRFU/SAp, F-91191 Gif-sur-Yvette Cedex, France

${ }^{16}$ Department of Physics \& Astronomy, Youngstown State University, Youngstown, OH 44555

${ }^{17}$ Université de Lyon 1, CRAL, Observatoire de Lyon, 9 av. Charles André, F-69230 Saint-Genis Laval; CNRS, UMR 5574; ENS de Lyon, France

${ }^{18}$ European Southern Observatory, Karl-Schwarzchild-Str. 2, D-85748 Garching, Germany

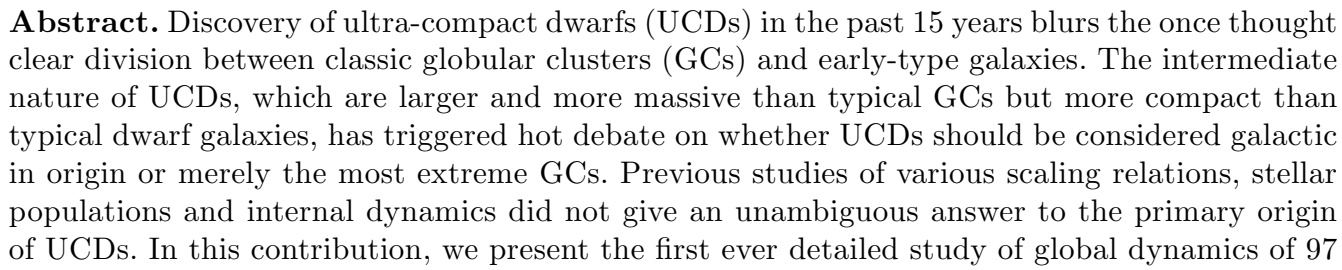


UCDs $\left(r_{\mathrm{h}} \gtrsim 10 \mathrm{pc}\right)$ associated with the central cD galaxy of the Virgo cluster, M87. We found that UCDs follow a different radial number density profile and different rotational properties from GCs. The orbital anisotropies of UCDs are tangentially-biased within $\sim 40 \mathrm{kpc}$ of M87 and become radially-biased with radius further out. In contrast, the blue GCs, which have similar median colors to our sample of UCDs, become more tangentially-biased at larger radii beyond $\sim 40 \mathrm{kpc}$. Our analysis suggests that most UCDs in M87 are not consistent with being merely the most luminous and extended examples of otherwise normal GCs. The radially-biased orbital structure of UCDs at large radii is in general agreement with the scenario that most UCDs originated from the tidally threshed dwarf galaxies.

Keywords. galaxies: clusters, globular clusters, galaxies: nuclei, galaxies: elliptical and lenticular, cD, galaxies: kinematics and dynamics

\section{Data and samples}

This work is devoted to a comparative study of the dynamical properties of M87 UCDs, GCs and surrounding dwarf ellipticals. Our samples of 97 confirmed UCDs and 911 confirmed GCs within the central $1.5^{\circ}$ of M87 are collected from both literature (e.g. Hanes et al. 2001; Strader et al. 2011) and our new observations. For new observations, we selected UCD and GC candidates for spectroscopic followup with Hectospec/MMT and $2 \mathrm{dF} / \mathrm{AAT}$ based on the high-sensitivity $\left(g_{\mathrm{lim}}=25.9 \mathrm{mag}\right.$ at $10 \sigma$ for point sources $)$ and high-resolution (FWHM $\sim 0.6^{\prime \prime}$ in $i$ band) $u^{*}$ griz imaging data from the Next Generation Virgo Cluster Survey (NGVS, Ferrarese et al. 2012).

The readers are referred to Zhang et al. (2015) for details of the samples and new observations. Briefly, our spectroscopic surveys of Virgo UCDs and GCs have been highly efficient, thanks to an unprecedentedly clean sample of Virgo UCD and GC candidates selected from NGVS. Our surveys covered nearly all of the area encompassed by one scale radius of the NFW dark matter halo toward the Virgo A subcluster $\left(2^{\circ} .143=0.617 \mathrm{Mpc}\right.$; McLaughlin 1999). Because the half-light radius $r_{\mathrm{h}, \mathrm{NGVS}}$ measurement for relatively faint sources is subject to large uncertainties, we only select UCDs with $g \leqslant 21.5$ mag and $r_{\mathrm{h}, \mathrm{NGVS}} \geqslant 11$ pc. In addition, sources with HST size measurement $r_{\mathrm{h}, \mathrm{HST}}>9.5 \mathrm{pc}$ are also included as UCDs, irrespective of their brightness. All the other confirmed Virgo compact clusters are regarded to be GCs. Overall, our sample of UCDs is expected to be $\sim 60 \%$ complete at $g_{0}<21.5 \mathrm{mag}$. The median $(g-i)_{0}$ color of our samples of UCDs and blue GCs are 0.75 and 0.74 respectively, and about $92 \%$ of our UCDs fall into the color range of the blue GCs. So we place additional emphasis on a comparison between properties of UCDs and blue GCs in this work.

\section{Results and discussion}

Surface number density profiles. Our UCD sample is $\sim 98 \%$ complete at $g_{0}<20.5$, which corresponds to $M_{g}<-10.6$. In Figure 1, we show the radial number density profiles of the 59 UCDs with $g_{0}<20.5 \mathrm{mag}$, together with profiles of the photometrically selected blue GCs, red GCs $\left(g_{0}<24\right.$ mag, Durrell et al. 2014) and the surrounding dE galaxies.

We adopted the Sérsic function to quantify (curves in Figure 1) the radial profiles of UCDs and GCs. Details about the profile fitting is described in Zhang et al. (2015). UCDs have a radial number density profile that is shallower than GCs in the inner $15^{\prime}$ and as steep as the red GCs at large radii. The surrounding dE galaxies have much flatter and extended number density profiles than UCDs and GCs. 


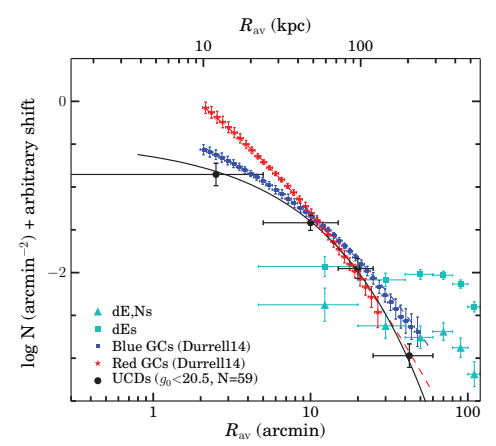

Figure 1. Radial number density profiles of UCDs (black), blue GCs (blue), red GCs (red), dE, Ns (cyan triangles) and all dE (cyan squares) galaxies. Overplotted on the data are the best-fit Sérsic profiles for UCDs and GCs. Note that radial profiles of the GCs have been vertically shifted down arbitrarily (2.1 for the blue and 1.7 for the red GCs) for comparison purpose.

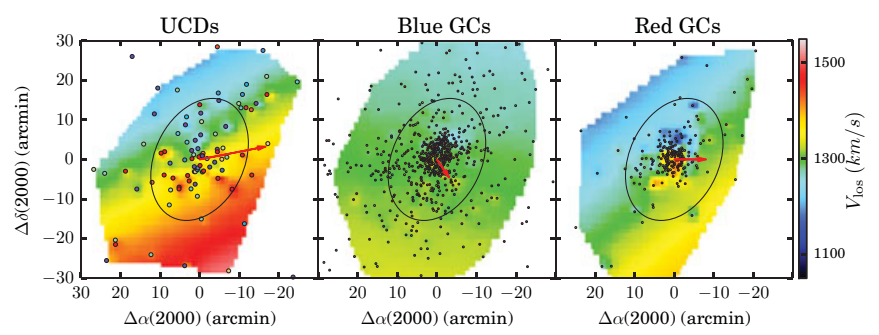

Figure 2. Spatial distribution of the UCDs (left), blue GCs (middle) and red GCs (right) are over plotted on their respective surface fitting (the color background) to the spatial distribution of line-of-sight velocities with the Kriging technique for the inner $30^{\prime}$ of M87. The black ellipses represent the stellar isophotes of M87 at $10 R_{\mathrm{e}}$, and the black solid line marks the photometric minor axis of M87 in each panel. The red arrows, with the length being proportional to the rotation amplitude, mark the direction of rotation axis from our global kinematics fitting of $v_{\text {los }}$ vs. PA to the inner $30^{\prime}$. The global kinematics fitting, which is primarily driven by the inner regions that contain most of the data points, matches the central velocity field from Kriging surface fitting. Among the three populations, the blue GCs seem to have an overall velocity field more aligned with the photometric major axis than the other two populations.

Velocity field. The UCDs and blue GCs have similar intrinsic velocity dispersion. The rotation amplitude of UCDs is more than 3 times stronger than that of the blue and red GCs. In addition, the overall velocity field of blue GCs is aligned with the photometric major axis of M87 much better than UCDs and the red GCs. Our test suggests that the probability of finding a rotation amplitude greater than or equal to the best-fit value for UCDs purely by chance is $\sim 2 \%$, the probability for blue GCs is $\sim 27 \%$, and $\sim 12 \%$ for the red GCs. The significantly stronger rotation of UCDs as compared to GCs suggests that UCDs are kinematically distinct from GCs.

Orbital anisotropies. To constrain the orbital anisotropy profiles $\beta_{r}$ of UCDs, blue and red GCs, we turn to the spherically symmetric Jeans equation. In determining $\beta_{r}$, we adopt the most recent determination of M87 mass profile by Zhu et al. (2014) based on made-to-measure modeling of over 900 M87 GCs. In addition, the surface number density profiles are de-projected to derive the 3D number density profiles which are used in solving the Jeans equation. Details of the Jeans modeling, including the functional form of $\beta_{r}$, can be found in Zhang et al. (2015). Basically, we used a maximum likelihood method to fit Jeans models to the observed $v_{\mathrm{los}, \mathrm{i}}$ as a function of geometric average radius $R_{i}$, by assuming that $v_{\mathrm{los}, \mathrm{i}}$ at $R_{i}$ follows a Gaussian distribution. The most probable model $\beta_{r}$ 


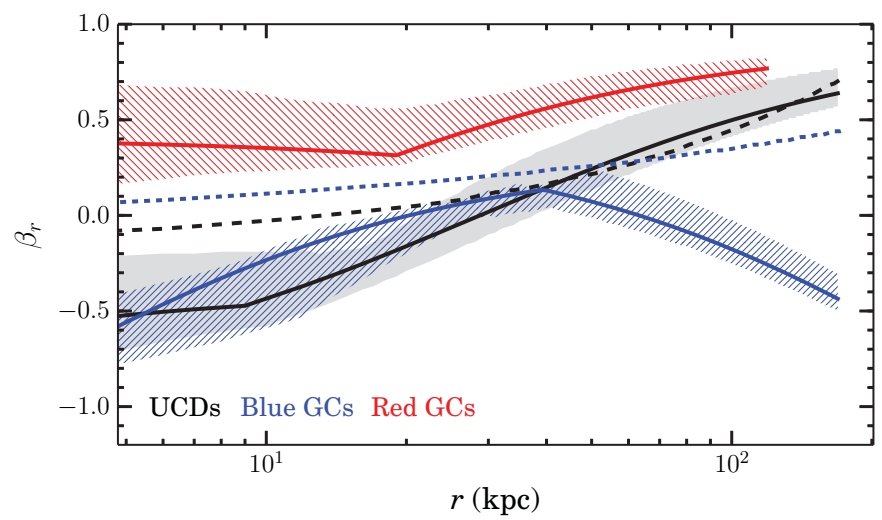

Figure 3. Variation of the anisotropy parameters as a function of the 3D radius. The profiles for UCDs, blue GCs, and red GCs are represented as black, blue, and red solid curves respectively. Following the same color code, the hatched regions of different styles mark the $68 \%$ confidence intervals for blue GCs, and red GCs. The grey shaded region marks the $68 \%$ confidence interval for the UCDs. The short dashed curves (black for UCDs, blue for blue GCs) represent the anisotropy profiles predicted by a universal relation between the number density slope and $\beta$ for relic high- $\sigma$ density peaks as found in cosmological simulations by Diemand et al. (2005).

profile for each population is taken as the fiducial one, and the $68 \%$ confidence intervals are determined by randomly resampling the real data sets, with $\sim 10 \%$ of data points being left out for each resample.

The determined $\beta_{r}$ profiles for UCDs, blue and red GCs are shown in Figure 3. The UCD system has an anisotropy profile that becomes more radial with radius, with $\beta_{r}$ being negative within the inner $\sim 40 \mathrm{kpc}$ and being positive beyond. The blue GCs have a radially increasing $\beta_{r}$ profile in the inner $\sim 40 \mathrm{kpc}$ but a radially decreasing profile at larger radii. Among the three populations, the red GCs exhibit the largest radially-biased orbital structure across the explored radius range.

Although being more negative in the innermost radii, the $\beta_{r}$ profile of UCDs determined from Jeans analysis is more or less consistent with the cosmological simulations of Diemand et al. (2005). In contrast, the blue GCs exhibit large deviation from the cosmological simulations. The significantly tangentially-biased orbital structure of UCDs at small radii can be partly attributed to a strong tidal transformation. The finding that the blue GCs are tangentially-biased, rather than radially-biased, at large radii may indicate that the blue GCs in the outer halo of M87 have not yet established an equilibrium state and is still in an early and active stage of assembly by continuously accreting the surrounding dwarf galaxies (e.g. Côté, Marzke \& West 1998).

\section{Conclusion}

We conclude that most UCDs in M87 are not consistent with being merely the most luminous and extended examples of otherwise normal GCs. The radially-biased orbital structure of UCDs at large radii is in general agreement with the scenario that UCDs are tidally stripped nuclei of dwarf galaxies. The distinct rotational properties of UCDs, as compared to GCs and the surrounding dE galaxies, indicate that the primary UCD progenitors do not necessarily resemble the present-day surviving dwarf galaxies.

\section{References}

Côté, P., Marzke, R. O., \& West, M. J. 1998, ApJ, 501, 554

Diemand, J., Madau, P., \& Moore, B. 2005, MNRAS, 364, 367 
Durrell, P. R., Côté, P., Peng, E. W., et al. 2014, ApJ, 794, 103

Ferrarese, L., Côté, P., Cuillandre, J. -C., et al. 2012, ApJS, 200, 4

Hanes, D. A., Côté, P., Bridges, T. J., et al. 2001, ApJ, 559, 812

Strader, J., Romanowsky, A. J., Brodie, J. P., et al. 2011, ApJS, 197, 33

Zhang, H. -X., Peng, E. W., Côté, P., et al. 2015, ApJ, in press, arXiv: 1501.03167

Zhu, L., Long, R. J., Mao, S., et al. 2014, ApJ, 792, 59 